\title{
How can we predict accurate electrochromic shifts for biochromophores? A case study on the photosynthetic reaction center
}

\author{
Abhishek Sirohiwal, ${ }^{a b}$ Frank Neese, ${ }^{a}$ Dimitrios A. Pantazis ${ }^{a *}$
}

${ }^{a}$ Max-Planck-Institut für Kohlenforschung, Kaiser-Wilhelm-Platz 1, 45470 Mülheim an der Ruhr, Germany.

${ }^{\mathrm{b}}$ Fakultät für Chemie und Biochemie, Ruhr-Universität Bochum, 44780 Bochum, Germany 
Table S1. The vertical excitation energies of the lowest excited state (i.e. $\mathrm{Q}_{y}$ ) using the QM/MM optimized geometries of the $\mathrm{RC}$ chromophores computed using canonical $\mathrm{CC} 2$ in the gas phase. Corresponding oscillator strengths $(f)$ are also shown. The major contributions in the canonical basis associated with the $\mathrm{Q}_{y}$ state are described.

\begin{tabular}{|c|c|c|c|c|}
\hline Chromophore & Energy (eV) & $f$ & Frontier Molecular Orbitals & $\%$ Contribution \\
\hline \multirow{4}{*}{$\mathrm{P}_{\mathrm{D} 1}$} & \multirow{3}{*}{2.126} & \multirow{3}{*}{0.19} & $\mathrm{HOMO} \rightarrow$ LUMO & 0.50 \\
\hline & & & $\mathrm{HOMO}^{-1} \rightarrow$ LUMO & 0.27 \\
\hline & & & HOMO-1 $\rightarrow$ LUMO+1 & 0.15 \\
\hline & \multirow{4}{*}{2.131} & \multirow{3}{*}{0.16} & HOMO $\rightarrow$ LUMO & 0.44 \\
\hline \multirow{3}{*}{$\mathrm{P}_{\mathrm{D} 2}$} & & & HOMO-1 $\rightarrow$ LUMO & 0.32 \\
\hline & & & HOMO-1 $\rightarrow$ LUMO+1 & 0.13 \\
\hline & & \multirow{3}{*}{0.21} & $\mathrm{HOMO} \rightarrow$ LUMO & 0.51 \\
\hline \multirow[t]{3}{*}{$\mathrm{Chl}_{\mathrm{D} 1}$} & \multirow[t]{3}{*}{2.128} & & HOMO- $1 \rightarrow$ LUMO & 0.26 \\
\hline & & & HOMO-1 $\rightarrow$ LUMO+1 & 0.14 \\
\hline & & \multirow{3}{*}{0.21} & $\mathrm{HOMO} \rightarrow$ LUMO & 0.53 \\
\hline \multirow[t]{2}{*}{$\mathrm{Chl}_{\mathrm{D} 2}$} & \multirow[t]{2}{*}{2.117} & & HOMO-1 $\rightarrow$ LUMO & 0.24 \\
\hline & & & HOMO-1 $\rightarrow$ LUMO+1 & 0.15 \\
\hline \multirow{2}{*}{$\mathrm{Pheo}_{\mathrm{D} 1}$} & \multirow{2}{*}{2.089} & \multirow{2}{*}{0.17} & HOMO $\rightarrow$ LUMO & 0.69 \\
\hline & & & $\mathrm{HOMO}-1 \rightarrow \mathrm{LUMO}+1$ & 0.25 \\
\hline \multirow{2}{*}{$\mathrm{PheO}_{\mathrm{D} 2}$} & \multirow{2}{*}{2.084} & \multirow{2}{*}{0.17} & $\mathrm{HOMO} \rightarrow$ LUMO & 0.69 \\
\hline & & & HOMO-1 $\rightarrow$ LUMO+1 & 0.25 \\
\hline
\end{tabular}

Table S2. The vertical excitation energies of the lowest excited state (i.e. $\mathrm{Q}_{y}$ ) using the QM/MM optimized geometries of the $\mathrm{RC}$ chromophores computed using canonical $\mathrm{CC} 2$ in the protein electrostatic environment. Corresponding oscillator strengths $(f)$ are also shown. The major contributions in the canonical basis associated with the $\mathrm{Q}_{y}$ state are described.

\begin{tabular}{|c|c|c|c|c|}
\hline Chromophore & Energy (eV) & $f$ & Frontier Molecular Orbitals & $\%$ Contribution \\
\hline \multirow{4}{*}{$\mathrm{P}_{\mathrm{D} 1}$} & \multirow{4}{*}{2.137} & \multirow{3}{*}{0.25} & $\mathrm{HOMO} \rightarrow \mathrm{LUMO}$ & 0.60 \\
\hline & & & HOMO-1 $\rightarrow$ LUMO & 0.18 \\
\hline & & & HOMO-1 $\rightarrow$ LUMO+1 & 0.15 \\
\hline & & \multirow{3}{*}{0.20} & $\mathrm{HOMO} \rightarrow$ LUMO & 0.54 \\
\hline \multirow{3}{*}{$\mathrm{P}_{\mathrm{D} 2}$} & \multirow[t]{3}{*}{2.134} & & HOMO-1 $\rightarrow$ LUMO & 0.24 \\
\hline & & & HOMO-1 $\rightarrow$ LUMO+1 & 0.14 \\
\hline & & \multirow{3}{*}{0.27} & $\mathrm{HOMO} \rightarrow$ LUMO & 0.56 \\
\hline \multirow[t]{3}{*}{$\mathrm{Ch}_{\mathrm{D} 1}$} & \multirow{3}{*}{2.098} & & HOMO- $1 \rightarrow$ LUMO & 0.23 \\
\hline & & & $\mathrm{HOMO}-1 \rightarrow \mathrm{LUMO}+1$ & 0.14 \\
\hline & & \multirow{3}{*}{0.25} & $\mathrm{HOMO} \rightarrow$ LUMO & 0.60 \\
\hline \multirow{2}{*}{$\mathrm{Chl}_{\mathrm{D} 2}$} & \multirow[t]{2}{*}{2.105} & & HOMO-1 $\rightarrow$ LUMO & 0.18 \\
\hline & & & $\mathrm{HOMO}-1 \rightarrow \mathrm{LUMO}+1$ & 0.15 \\
\hline \multirow{2}{*}{$\mathrm{PheO}_{\mathrm{D} 1}$} & \multirow{2}{*}{2.114} & \multirow{2}{*}{0.19} & $\mathrm{HOMO} \rightarrow$ LUMO & 0.66 \\
\hline & & & $\mathrm{HOMO}-1 \rightarrow \mathrm{LUMO}+1$ & 0.22 \\
\hline \multirow{2}{*}{$\mathrm{PheO}_{\mathrm{D} 2}$} & \multirow{2}{*}{2.099} & \multirow[t]{2}{*}{0.18} & $\mathrm{HOMO} \rightarrow$ LUMO & 0.61 \\
\hline & & & HOMO-1 $\rightarrow$ LUMO+1 & 0.21 \\
\hline
\end{tabular}


Table S3. The vertical excitation energies of the lowest excited state (i.e. $\mathrm{Q}_{y}$ ) using the QM/MM optimized geometries of the RC chromophores computed using SOS-CC2 in the gas phase. Corresponding oscillator strengths $(f)$ are also shown. The major contributions in the canonical basis associated with the $\mathrm{Q}_{y}$ state are described.

\begin{tabular}{|c|c|c|c|c|}
\hline Chromophore & Energy (eV) & $f$ & Frontier Molecular Orbitals & $\%$ Contribution \\
\hline$P_{D 1}$ & 2.022 & 0.21 & $\begin{aligned} \mathrm{HOMO} & \rightarrow \text { LUMO } \\
\mathrm{HOMO}-1 & \rightarrow \text { LUMO+1 }\end{aligned}$ & $\begin{array}{l}0.72 \\
0.19\end{array}$ \\
\hline $\mathrm{P}_{\mathrm{D} 2}$ & 2.027 & 0.19 & $\begin{aligned} \text { HOMO } & \rightarrow \text { LUMO } \\
\text { HOMO-1 } & \rightarrow \text { LUMO+1 }\end{aligned}$ & $\begin{array}{l}0.72 \\
0.21\end{array}$ \\
\hline $\mathrm{Chl}_{\mathrm{D} 1}$ & 2.027 & 0.23 & $\begin{aligned} \mathrm{HOMO} & \rightarrow \text { LUMO } \\
\mathrm{HOMO}-1 & \rightarrow \text { LUMO+1 }\end{aligned}$ & $\begin{array}{l}0.73 \\
0.19\end{array}$ \\
\hline $\mathrm{Ch}_{\mathrm{D} 2}$ & 2.037 & 0.22 & $\begin{aligned} \mathrm{HOMO} & \rightarrow \text { LUMO } \\
\mathrm{HOMO}-1 & \rightarrow \text { LUMO+1 }\end{aligned}$ & $\begin{array}{l}0.72 \\
0.19\end{array}$ \\
\hline $\mathrm{Pheo}_{\mathrm{D} 1}$ & 2.002 & 0.17 & $\begin{aligned} \mathrm{HOMO} & \rightarrow \text { LUMO } \\
\mathrm{HOMO}-1 & \rightarrow \text { LUMO+1 }\end{aligned}$ & $\begin{array}{l}0.69 \\
0.24\end{array}$ \\
\hline $\mathrm{PheO}_{\mathrm{D} 2}$ & 2.004 & 0.17 & $\begin{aligned} \mathrm{HOMO} & \rightarrow \text { LUMO } \\
\mathrm{HOMO}-1 & \rightarrow \text { LUMO+1 }\end{aligned}$ & $\begin{array}{l}0.69 \\
0.24\end{array}$ \\
\hline
\end{tabular}

Table S4. The vertical excitation energies of the lowest excited state (i.e. $\mathrm{Q}_{y}$ ) using the QM/MM optimized geometries of the RC chromophores computed using SOS-CC2 in the protein electrostatic environment. Corresponding oscillator strengths $(f)$ are also shown. The major contributions in the canonical basis associated with the $\mathrm{Q}_{y}$ state are described.

\begin{tabular}{|c|c|c|c|c|}
\hline Chromophore & Energy (eV) & $f$ & Frontier Molecular Orbitals & $\%$ Contribution \\
\hline$P_{D 1}$ & 2.004 & 0.26 & $\begin{aligned} \text { HOMO } & \rightarrow \text { LUMO } \\
\text { HOMO-1 } & \rightarrow \text { LUMO+1 }\end{aligned}$ & $\begin{array}{l}0.76 \\
0.18\end{array}$ \\
\hline $\mathrm{P}_{\mathrm{D} 2}$ & 2.012 & 0.24 & $\begin{aligned} \mathrm{HOMO} & \rightarrow \text { LUMO } \\
\mathrm{HOMO}-1 & \rightarrow \text { LUMO+1 }\end{aligned}$ & $\begin{array}{l}0.76 \\
0.19\end{array}$ \\
\hline $\mathrm{Chl}_{\mathrm{D} 1}$ & 1.961 & 0.29 & $\begin{aligned} \text { HOMO } & \rightarrow \text { LUMO } \\
\text { HOMO-1 } & \rightarrow \text { LUMO+1 }\end{aligned}$ & $\begin{array}{l}0.77 \\
0.17\end{array}$ \\
\hline $\mathrm{Ch}_{\mathrm{D} 2}$ & 2.010 & 0.25 & $\begin{aligned} \mathrm{HOMO} & \rightarrow \text { LUMO } \\
\mathrm{HOMO}-1 & \rightarrow \text { LUMO+1 }\end{aligned}$ & $\begin{array}{l}0.75 \\
0.18\end{array}$ \\
\hline Pheod $1_{1}$ & 2.154 & 0.14 & $\begin{aligned} \mathrm{HOMO} & \rightarrow \text { LUMO } \\
\mathrm{HOMO}-1 & \rightarrow \text { LUMO+1 }\end{aligned}$ & $\begin{array}{l}0.68 \\
0.23\end{array}$ \\
\hline $\mathrm{Pheo}_{\mathrm{D} 2}$ & 2.171 & 0.14 & $\begin{aligned} \text { HOMO } & \rightarrow \text { LUMO } \\
\text { HOMO-1 } & \rightarrow \text { LUMO+1 }\end{aligned}$ & $\begin{array}{l}0.67 \\
0.24\end{array}$ \\
\hline
\end{tabular}


Table S5. The vertical excitation energies of the lowest excited state (i.e. $\mathrm{Q}_{y}$ ) using the QM/MM optimized geometries of the RC chromophores computed using SCS-CC2 in the gas phase. Corresponding oscillator strengths $(f)$ are also shown. The major contributions in the canonical basis associated with the $\mathrm{Q}_{y}$ state are described.

\begin{tabular}{|c|c|c|c|c|}
\hline Chromophore & Energy (eV) & $f$ & Frontier Molecular Orbitals & $\%$ Contribution \\
\hline $\mathrm{P}_{\mathrm{D} 1}$ & 2.064 & 0.23 & $\begin{aligned} \mathrm{HOMO} & \rightarrow \text { LUMO } \\
\mathrm{HOMO}-1 & \rightarrow \text { LUMO }+1\end{aligned}$ & $\begin{array}{l}0.75 \\
0.20\end{array}$ \\
\hline $\mathrm{P}_{\mathrm{D} 2}$ & 2.071 & 0.20 & $\begin{aligned} \mathrm{HOMO} & \rightarrow \text { LUMO } \\
\mathrm{HOMO}-1 & \rightarrow \text { LUMO+1 }\end{aligned}$ & $\begin{array}{l}0.74 \\
0.21\end{array}$ \\
\hline $\mathrm{Chl}_{\mathrm{D} 1}$ & 2.068 & 0.24 & $\begin{aligned} \mathrm{HOMO} & \rightarrow \mathrm{LUMO} \\
\mathrm{HOMO}-1 & \rightarrow \text { LUMO+1 }\end{aligned}$ & $\begin{array}{l}0.75 \\
0.19\end{array}$ \\
\hline $\mathrm{Chl}_{\mathrm{D} 2}$ & 2.071 & 0.24 & $\begin{aligned} \mathrm{HOMO} & \rightarrow \text { LUMO } \\
\mathrm{HOMO}-1 & \rightarrow \text { LUMO+1 }\end{aligned}$ & $\begin{array}{l}0.75 \\
0.20\end{array}$ \\
\hline $\mathrm{Pheo}_{\mathrm{D} 1}$ & 2.023 & 0.17 & $\begin{aligned} \mathrm{HOMO} & \rightarrow \text { LUMO } \\
\mathrm{HOMO}-1 & \rightarrow \text { LUMO+1 }\end{aligned}$ & $\begin{array}{l}0.71 \\
0.25\end{array}$ \\
\hline $\mathrm{PheO}_{\mathrm{D} 2}$ & 2.023 & 0.17 & $\begin{aligned} \mathrm{HOMO} & \rightarrow \mathrm{LUMO} \\
\mathrm{HOMO}-1 & \rightarrow \text { LUMO+1 }\end{aligned}$ & $\begin{array}{l}0.71 \\
0.25\end{array}$ \\
\hline
\end{tabular}

Table S6. The vertical excitation energies of the lowest excited state (i.e. $\mathrm{Q}_{y}$ ) using the QM/MM optimized geometries of the RC chromophores computed using SCS-CC2 in the protein electrostatic environment. Corresponding oscillator strengths $(f)$ are also shown. The major contributions in the canonical basis associated with the $\mathrm{Q}_{y}$ state are described.

\begin{tabular}{|c|c|c|c|c|}
\hline Chromophore & Energy (eV) & $f$ & Frontier Molecular Orbitals & $\%$ Contribution \\
\hline$P_{D 1}$ & 2.051 & 0.27 & $\begin{aligned} \mathrm{HOMO} & \rightarrow \text { LUMO } \\
\mathrm{HOMO}-1 & \rightarrow \text { LUMO+1 }\end{aligned}$ & $\begin{array}{l}0.77 \\
0.18\end{array}$ \\
\hline $\mathrm{P}_{\mathrm{D} 2}$ & 2.058 & 0.24 & $\begin{aligned} \text { HOMO } & \rightarrow \text { LUMO } \\
\text { HOMO-1 } & \rightarrow \text { LUMO+1 }\end{aligned}$ & $\begin{array}{l}0.76 \\
0.19\end{array}$ \\
\hline $\mathrm{Chl}_{\mathrm{D} 1}$ & 2.011 & 0.30 & $\begin{aligned} \mathrm{HOMO} & \rightarrow \text { LUMO } \\
\mathrm{HOMO}-1 & \rightarrow \text { LUMO+1 }\end{aligned}$ & $\begin{array}{l}0.78 \\
0.17\end{array}$ \\
\hline $\mathrm{Ch}_{\mathrm{D} 2}$ & 2.046 & 0.27 & $\begin{aligned} \mathrm{HOMO} & \rightarrow \text { LUMO } \\
\mathrm{HOMO}-1 & \rightarrow \text { LUMO+1 }\end{aligned}$ & $\begin{array}{l}0.77 \\
0.18\end{array}$ \\
\hline $\mathrm{Pheo}_{\mathrm{D} 1}$ & 2.134 & 0.17 & $\begin{aligned} \mathrm{HOMO} & \rightarrow \text { LUMO } \\
\mathrm{HOMO}-1 & \rightarrow \text { LUMO+1 }\end{aligned}$ & $\begin{array}{l}0.71 \\
0.24\end{array}$ \\
\hline $\mathrm{PheO}_{\mathrm{D} 2}$ & 2.142 & 0.17 & $\begin{aligned} \mathrm{HOMO} & \rightarrow \text { LUMO } \\
\mathrm{HOMO}-1 & \rightarrow \text { LUMO+1 }\end{aligned}$ & $\begin{array}{l}0.71 \\
0.24\end{array}$ \\
\hline
\end{tabular}


Table S7. The vertical excitation energies of the lowest excited state (i.e. $\mathrm{Q}_{y}$ ) using the QM/MM optimized geometries of the $\mathrm{RC}$ chromophores computed using $\mathrm{ADC}(2)$ in the gas phase. Corresponding oscillator strengths $(f)$ are also shown. The major contributions in the canonical basis associated with the $\mathrm{Q}_{y}$ state are described.

\begin{tabular}{|c|c|c|c|c|}
\hline Chromophore & Energy (eV) & $f$ & Frontier Molecular Orbitals & $\%$ Contribution \\
\hline \multirow{4}{*}{$\mathrm{P}_{\mathrm{D} 1}$} & \multirow{3}{*}{1.887} & \multirow{3}{*}{0.21} & $\mathrm{HOMO} \rightarrow \mathrm{LUMO}$ & 0.46 \\
\hline & & & HOMO-1 $\rightarrow$ LUMO & 0.37 \\
\hline & & & $\mathrm{HOMO}-1 \rightarrow \mathrm{LUMO}+1$ & 0.07 \\
\hline & \multirow{4}{*}{1.898} & \multirow{4}{*}{0.18} & $\mathrm{HOMO} \rightarrow$ LUMO & 0.45 \\
\hline \multirow{3}{*}{$\mathrm{P}_{\mathrm{D} 2}$} & & & HOMO-1 $\rightarrow$ LUMO & 0.36 \\
\hline & & & $\mathrm{HOMO} \rightarrow \mathrm{LUMO}+1$ & 0.07 \\
\hline & & & $\mathrm{HOMO} \rightarrow \mathrm{LUMO}$ & 0.51 \\
\hline \multirow[t]{3}{*}{$\mathrm{Chl}_{\mathrm{D} 1}$} & \multirow[t]{3}{*}{1.896} & \multirow[t]{3}{*}{0.24} & $\mathrm{HOMO}-1 \rightarrow$ LUMO & 0.32 \\
\hline & & & $\mathrm{HOMO}-1 \rightarrow \mathrm{LUMO}+1$ & 0.08 \\
\hline & & & $\mathrm{HOMO} \rightarrow$ LUMO & 0.57 \\
\hline \multirow[t]{2}{*}{$\mathrm{Ch}_{\mathrm{D} 2}$} & \multirow{2}{*}{1.883} & \multirow[t]{2}{*}{0.25} & HOMO-1 $\rightarrow$ LUMO & 0.26 \\
\hline & & & $\mathrm{HOMO}-1 \rightarrow \mathrm{LUMO}+1$ & 0.09 \\
\hline \multirow{2}{*}{$\mathrm{Pheo}_{\mathrm{D} 1}$} & \multirow{2}{*}{1.873} & \multirow{2}{*}{0.23} & $\mathrm{HOMO} \rightarrow$ LUMO & 0.74 \\
\hline & & & $\mathrm{HOMO}-1 \rightarrow \mathrm{LUMO}+1$ & 0.17 \\
\hline \multirow{2}{*}{$\mathrm{PheO}_{\mathrm{D} 2}$} & \multirow[t]{2}{*}{1.863} & \multirow[t]{2}{*}{0.23} & $\mathrm{HOMO} \rightarrow$ LUMO & 0.74 \\
\hline & & & $\mathrm{HOMO}-1 \rightarrow \mathrm{LUMO}+1$ & 0.16 \\
\hline
\end{tabular}

Table S8. The vertical excitation energies of the lowest excited state (i.e. $\mathrm{Q}_{y}$ ) using the QM/MM optimized geometries of the $\mathrm{RC}$ chromophores computed using $\mathrm{ADC}(2)$ in the protein electrostatic environment. Corresponding oscillator strengths $(f)$ are also shown. The major contributions in the canonical basis associated with the $\mathrm{Q}_{y}$ state are described.

\begin{tabular}{|c|c|c|c|c|}
\hline Chromophore & Energy (eV) & $f$ & Frontier Molecular Orbitals & $\%$ Contribution \\
\hline \multirow{4}{*}{$\mathrm{P}_{\mathrm{D} 1}$} & \multirow{4}{*}{1.920} & \multirow{3}{*}{0.28} & $\mathrm{HOMO} \rightarrow \mathrm{LUMO}$ & 0.62 \\
\hline & & & HOMO-1 $\rightarrow$ LUMO & 0.22 \\
\hline & & & $\mathrm{HOMO}-1 \rightarrow \mathrm{LUMO}+1$ & 0.09 \\
\hline & & & $\mathrm{HOMO} \rightarrow$ LUMO & 0.59 \\
\hline \multirow[t]{3}{*}{$\mathrm{P}_{\mathrm{D} 2}$} & \multirow{3}{*}{1.918} & \multirow{3}{*}{0.25} & $\mathrm{HOMO}^{-1} \rightarrow$ LUMO & 0.24 \\
\hline & & & $\mathrm{HOMO}-1 \rightarrow \mathrm{LUMO}+1$ & 0.08 \\
\hline & & & $\mathrm{HOMO} \rightarrow$ LUMO & 0.53 \\
\hline \multirow[t]{3}{*}{$\mathrm{Ch}_{\mathrm{D} 1}$} & \multirow[t]{3}{*}{1.878} & \multirow[t]{3}{*}{0.29} & HOMO-1 $\rightarrow$ LUMO & 0.31 \\
\hline & & & $\mathrm{HOMO}-1 \rightarrow \mathrm{LUMO}+1$ & 0.08 \\
\hline & & & $\mathrm{HOMO} \rightarrow$ LUMO & 0.65 \\
\hline \multirow[t]{2}{*}{$\mathrm{Ch}_{\mathrm{D} 2}$} & \multirow[t]{2}{*}{1.874} & \multirow{2}{*}{0.29} & HOMO-1 $\rightarrow$ LUMO & 0.19 \\
\hline & & & $\mathrm{HOMO}-1 \rightarrow \mathrm{LUMO}+1$ & 0.09 \\
\hline \multirow{2}{*}{ Pheod $1_{1}$} & \multirow{2}{*}{1.916} & \multirow{2}{*}{0.23} & $\mathrm{HOMO} \rightarrow$ LUMO & 0.72 \\
\hline & & & $\mathrm{HOMO}-1 \rightarrow \mathrm{LUMO}+1$ & 0.14 \\
\hline \multirow{3}{*}{$\mathrm{PheO}_{\mathrm{D} 2}$} & \multirow{3}{*}{1.897} & \multirow{3}{*}{0.22} & $\mathrm{HOMO} \rightarrow$ LUMO & 0.67 \\
\hline & & & HOMO-1 $\rightarrow$ LUMO & 0.14 \\
\hline & & & $\mathrm{HOMO}-1 \rightarrow \mathrm{LUMO}+1$ & 0.13 \\
\hline
\end{tabular}


Table S9. The vertical excitation energies of the lowest excited state (i.e. $\mathrm{Q}_{y}$ ) using the QM/MM optimized geometries of the RC chromophores computed using SOS-ADC(2) in the gas phase. Corresponding oscillator strengths $(f)$ are also shown. The major contributions in the canonical basis associated with the $\mathrm{Q}_{y}$ state are described.

\begin{tabular}{|c|c|c|c|c|}
\hline Chromophore & Energy (eV) & $f$ & Frontier Molecular Orbitals & $\%$ Contribution \\
\hline$P_{D 1}$ & 1.936 & 0.24 & $\begin{aligned} \mathrm{HOMO} & \rightarrow \text { LUMO } \\
\mathrm{HOMO}-1 & \rightarrow \text { LUMO+1 }\end{aligned}$ & $\begin{array}{l}0.73 \\
0.16\end{array}$ \\
\hline $\mathrm{P}_{\mathrm{D} 2}$ & 1.940 & 0.21 & $\begin{aligned} \text { HOMO } & \rightarrow \text { LUMO } \\
\text { HOMO-1 } & \rightarrow \text { LUMO+1 }\end{aligned}$ & $\begin{array}{l}0.73 \\
0.18\end{array}$ \\
\hline $\mathrm{Ch}_{\mathrm{D} 1}$ & 1.942 & 0.26 & $\begin{aligned} \mathrm{HOMO} & \rightarrow \text { LUMO } \\
\mathrm{HOMO}-1 & \rightarrow \text { LUMO+1 }\end{aligned}$ & $\begin{array}{l}0.74 \\
0.16\end{array}$ \\
\hline $\mathrm{Ch}_{\mathrm{D} 2}$ & 1.952 & 0.24 & $\begin{aligned} \mathrm{HOMO} & \rightarrow \text { LUMO } \\
\mathrm{HOMO}-1 & \rightarrow \text { LUMO+1 }\end{aligned}$ & $\begin{array}{l}0.73 \\
0.16\end{array}$ \\
\hline $\mathrm{Pheo}_{\mathrm{D} 1}$ & 1.937 & 0.19 & $\begin{aligned} \mathrm{HOMO} & \rightarrow \text { LUMO } \\
\mathrm{HOMO}-1 & \rightarrow \text { LUMO }+1\end{aligned}$ & $\begin{array}{l}0.72 \\
0.21\end{array}$ \\
\hline $\mathrm{PheO}_{\mathrm{D} 2}$ & 1.939 & 0.19 & $\begin{aligned} \mathrm{HOMO} & \rightarrow \text { LUMO } \\
\mathrm{HOMO}-1 & \rightarrow \text { LUMO+1 }\end{aligned}$ & $\begin{array}{l}0.72 \\
0.21\end{array}$ \\
\hline
\end{tabular}

Table S10. The vertical excitation energies of the lowest excited state (i.e. $\mathrm{Q}_{y}$ ) using the QM/MM optimized geometries of the RC chromophores computed using SOS-ADC(2) in the protein electrostatic environment. Corresponding oscillator strengths $(f)$ are also shown. The major contributions in the canonical basis associated with the $\mathrm{Q}_{y}$ state are described.

\begin{tabular}{|c|c|c|c|c|}
\hline Chromophore & Energy (eV) & $f$ & Frontier Molecular Orbitals & $\%$ Contribution \\
\hline$P_{D 1}$ & 1.924 & 0.29 & $\begin{aligned} \text { HOMO } & \rightarrow \text { LUMO } \\
\text { HOMO-1 } & \rightarrow \text { LUMO+1 }\end{aligned}$ & $\begin{array}{l}0.78 \\
0.15\end{array}$ \\
\hline $\mathrm{P}_{\mathrm{D} 2}$ & 1.933 & 0.27 & $\begin{aligned} \mathrm{HOMO} & \rightarrow \text { LUMO } \\
\mathrm{HOMO}-1 & \rightarrow \text { LUMO+1 }\end{aligned}$ & $\begin{array}{l}0.79 \\
0.16\end{array}$ \\
\hline $\mathrm{Chl}_{\mathrm{D} 1}$ & 1.879 & 0.33 & $\begin{aligned} \text { HOMO } & \rightarrow \text { LUMO } \\
\text { HOMO-1 } & \rightarrow \text { LUMO+1 }\end{aligned}$ & $\begin{array}{l}0.79 \\
0.14\end{array}$ \\
\hline $\mathrm{Ch}_{\mathrm{D} 2}$ & 1.926 & 0.28 & $\begin{aligned} \mathrm{HOMO} & \rightarrow \text { LUMO } \\
\mathrm{HOMO}-1 & \rightarrow \text { LUMO+1 }\end{aligned}$ & $\begin{array}{l}0.77 \\
0.15\end{array}$ \\
\hline Pheod $1_{1}$ & 2.098 & 0.16 & $\begin{aligned} \mathrm{HOMO} & \rightarrow \text { LUMO } \\
\mathrm{HOMO}-1 & \rightarrow \text { LUMO+1 }\end{aligned}$ & $\begin{array}{l}0.69 \\
0.21\end{array}$ \\
\hline $\mathrm{PheO}_{\mathrm{D} 2}$ & 2.116 & 0.15 & $\begin{aligned} \mathrm{HOMO} & \rightarrow \text { LUMO } \\
\mathrm{HOMO}-1 & \rightarrow \text { LUMO+1 }\end{aligned}$ & $\begin{array}{l}0.67 \\
0.21\end{array}$ \\
\hline
\end{tabular}


Table S11. The vertical excitation energies of the lowest excited state (i.e. $\mathrm{Q}_{y}$ ) using the QM/MM optimized geometries of the RC chromophores computed using SCS-ADC(2) in the gas phase. Corresponding oscillator strengths $(f)$ are also shown. The major contributions in the canonical basis associated with the $\mathrm{Q}_{y}$ state are described.

\begin{tabular}{|c|c|c|c|c|}
\hline Chromophore & Energy (eV) & $f$ & Frontier Molecular Orbitals & $\%$ Contribution \\
\hline $\mathrm{P}_{\mathrm{D} 1}$ & 1.946 & 0.26 & $\begin{aligned} \mathrm{HOMO} & \rightarrow \text { LUMO } \\
\mathrm{HOMO}-1 & \rightarrow \text { LUMO }+1\end{aligned}$ & $\begin{array}{l}0.77 \\
0.16\end{array}$ \\
\hline $\mathrm{P}_{\mathrm{D} 2}$ & 1.952 & 0.23 & $\begin{aligned} \mathrm{HOMO} & \rightarrow \text { LUMO } \\
\mathrm{HOMO}-1 & \rightarrow \text { LUMO+1 }\end{aligned}$ & $\begin{array}{l}0.77 \\
0.18\end{array}$ \\
\hline $\mathrm{Chl}_{\mathrm{D} 1}$ & 1.952 & 0.28 & $\begin{aligned} \mathrm{HOMO} & \rightarrow \mathrm{LUMO} \\
\mathrm{HOMO}-1 & \rightarrow \text { LUMO+1 }\end{aligned}$ & $\begin{array}{l}0.78 \\
0.16\end{array}$ \\
\hline $\mathrm{Chl}_{\mathrm{D} 2}$ & 1.955 & 0.27 & $\begin{aligned} \mathrm{HOMO} & \rightarrow \text { LUMO } \\
\mathrm{HOMO}-1 & \rightarrow \text { LUMO+1 }\end{aligned}$ & $\begin{array}{l}0.78 \\
0.16\end{array}$ \\
\hline $\mathrm{Pheo}_{\mathrm{D} 1}$ & 1.930 & 0.21 & $\begin{aligned} \mathrm{HOMO} & \rightarrow \text { LUMO } \\
\mathrm{HOMO}-1 & \rightarrow \text { LUMO+1 }\end{aligned}$ & $\begin{array}{l}0.75 \\
0.20\end{array}$ \\
\hline $\mathrm{PheO}_{\mathrm{D} 2}$ & 1.929 & 0.21 & $\begin{aligned} \mathrm{HOMO} & \rightarrow \mathrm{LUMO} \\
\mathrm{HOMO}-1 & \rightarrow \text { LUMO+1 }\end{aligned}$ & $\begin{array}{l}0.75 \\
0.20\end{array}$ \\
\hline
\end{tabular}

Table S12. The vertical excitation energies of the lowest excited state (i.e. $\mathrm{Q}_{y}$ ) using the QM/MM optimized geometries of the RC chromophores computed using SCS-ADC(2) in the protein electrostatic environment. Corresponding oscillator strengths $(f)$ are also shown. The major contributions in the canonical basis associated with the $\mathrm{Q}_{y}$ state are described.

\begin{tabular}{|c|c|c|c|c|}
\hline Chromophore & Energy (eV) & $f$ & Frontier Molecular Orbitals & $\%$ Contribution \\
\hline $\mathrm{P}_{\mathrm{D} 1}$ & 1.940 & 0.31 & $\begin{aligned} \mathrm{HOMO} & \rightarrow \text { LUMO } \\
\mathrm{HOMO}-1 & \rightarrow \text { LUMO+1 }\end{aligned}$ & $\begin{array}{l}0.81 \\
0.14\end{array}$ \\
\hline $\mathrm{P}_{\mathrm{D} 2}$ & 1.947 & 0.29 & $\begin{aligned} \mathrm{HOMO} & \rightarrow \text { LUMO } \\
\mathrm{HOMO}-1 & \rightarrow \text { LUMO+1 }\end{aligned}$ & $\begin{array}{l}0.80 \\
0.15\end{array}$ \\
\hline $\mathrm{Chl}_{\mathrm{D} 1}$ & 1.898 & 0.35 & $\begin{aligned} \mathrm{HOMO} & \rightarrow \text { LUMO } \\
\mathrm{HOMO}-1 & \rightarrow \text { LUMO+1 }\end{aligned}$ & $\begin{array}{l}0.81 \\
0.14\end{array}$ \\
\hline $\mathrm{Ch}_{\mathrm{D} 2}$ & 1.930 & 0.31 & $\begin{aligned} \mathrm{HOMO} & \rightarrow \text { LUMO } \\
\mathrm{HOMO}-1 & \rightarrow \text { LUMO+1 }\end{aligned}$ & $\begin{array}{l}0.81 \\
0.14\end{array}$ \\
\hline Pheod $1_{1}$ & 2.059 & 0.19 & $\begin{aligned} \mathrm{HOMO} & \rightarrow \text { LUMO } \\
\mathrm{HOMO}-1 & \rightarrow \text { LUMO+1 }\end{aligned}$ & $\begin{array}{l}0.75 \\
0.20\end{array}$ \\
\hline $\mathrm{Pheo}_{\mathrm{D} 2}$ & 2.070 & 0.20 & $\begin{aligned} \text { HOMO } & \rightarrow \text { LUMO } \\
\text { HOMO-1 } & \rightarrow \text { LUMO+1 }\end{aligned}$ & $\begin{array}{l}0.75 \\
0.20\end{array}$ \\
\hline
\end{tabular}


Table S13. The vertical excitation energies of the lowest excited state (i.e. $\mathrm{Q}_{y}$ ) using the QM/MM optimized geometries of the RC chromophores computed using DLPNO-STEOM-CCSD in the gas phase. Corresponding oscillator strengths $(f)$ are also shown. The major contributions in the canonical basis associated with the $\mathrm{Q}_{y}$ state are described.

\begin{tabular}{|c|c|c|c|c|}
\hline Chromophore & Energy (eV) & $f$ & Frontier Molecular Orbitals & $\%$ Contribution \\
\hline$P_{D 1}$ & 1.633 & 0.22 & $\begin{aligned} \text { HOMO } & \rightarrow \text { LUMO } \\
\text { HOMO-1 } & \rightarrow \text { LUMO+1 }\end{aligned}$ & $\begin{array}{l}0.69 \\
0.18\end{array}$ \\
\hline $\mathrm{P}_{\mathrm{D} 2}$ & 1.635 & 0.20 & $\begin{aligned} \mathrm{HOMO} & \rightarrow \text { LUMO } \\
\mathrm{HOMO}-1 & \rightarrow \text { LUMO+1 }\end{aligned}$ & $\begin{array}{l}0.69 \\
0.19\end{array}$ \\
\hline $\mathrm{Chl}_{\mathrm{D} 1}$ & 1.642 & 0.23 & $\begin{aligned} \text { HOMO } & \rightarrow \text { LUMO } \\
\text { HOMO-1 } & \rightarrow \text { LUMO+1 }\end{aligned}$ & $\begin{array}{l}0.69 \\
0.17\end{array}$ \\
\hline $\mathrm{Ch}_{\mathrm{D} 2}$ & 1.649 & 0.23 & $\begin{aligned} \mathrm{HOMO} & \rightarrow \mathrm{LUMO} \\
\mathrm{HOMO}-1 & \rightarrow \text { LUMO+1 }\end{aligned}$ & $\begin{array}{l}0.68 \\
0.17\end{array}$ \\
\hline Pheo $_{D 1}$ & 1.601 & 0.15 & $\begin{aligned} \mathrm{HOMO} & \rightarrow \text { LUMO } \\
\mathrm{HOMO}-1 & \rightarrow \text { LUMO+1 }\end{aligned}$ & $\begin{array}{l}0.66 \\
0.25\end{array}$ \\
\hline $\mathrm{PheO}_{\mathrm{D} 2}$ & 1.591 & 0.16 & $\begin{aligned} \text { HOMO } & \rightarrow \text { LUMO } \\
\text { HOMO-1 } & \rightarrow \text { LUMO+1 }\end{aligned}$ & $\begin{array}{l}0.66 \\
0.25\end{array}$ \\
\hline
\end{tabular}

Table S14. The vertical excitation energies of the lowest excited state (i.e. $\mathrm{Q}_{y}$ ) using the QM/MM optimized geometries of the RC chromophores computed using DLPNO-STEOM-CCSD in the protein electrostatic environment. Corresponding oscillator strengths $(f)$ are also shown. The major contributions in the canonical basis associated with the $\mathrm{Q}_{y}$ state are described.

\begin{tabular}{|c|c|c|c|c|}
\hline Chromophore & Energy (eV) & $f$ & Frontier Molecular Orbitals & $\%$ Contribution \\
\hline$P_{D 1}$ & 1.613 & 0.27 & $\begin{aligned} \mathrm{HOMO} & \rightarrow \text { LUMO } \\
\mathrm{HOMO}-1 & \rightarrow \text { LUMO+1 }\end{aligned}$ & $\begin{array}{l}0.73 \\
0.17\end{array}$ \\
\hline $\mathrm{P}_{\mathrm{D} 2}$ & 1.620 & 0.26 & $\begin{aligned} \text { HOMO } & \rightarrow \text { LUMO } \\
\text { HOMO-1 } & \rightarrow \text { LUMO+1 }\end{aligned}$ & $\begin{array}{l}0.74 \\
0.18\end{array}$ \\
\hline $\mathrm{Chl}_{\mathrm{D} 1}$ & 1.575 & 0.30 & $\begin{aligned} \mathrm{HOMO} & \rightarrow \text { LUMO } \\
\mathrm{HOMO}-1 & \rightarrow \text { LUMO+1 }\end{aligned}$ & $\begin{array}{l}0.74 \\
0.16\end{array}$ \\
\hline $\mathrm{Chl}_{\mathrm{D} 2}$ & 1.624 & 0.26 & $\begin{aligned} \text { HOMO } & \rightarrow \text { LUMO } \\
\text { HOMO-1 } & \rightarrow \text { LUMO+1 }\end{aligned}$ & $\begin{array}{l}0.71 \\
0.17\end{array}$ \\
\hline $\mathrm{Pheo}_{\mathrm{D} 1}$ & 1.743 & 0.13 & $\begin{aligned} \mathrm{HOMO} & \rightarrow \text { LUMO } \\
\mathrm{HOMO}-1 & \rightarrow \text { LUMO+1 }\end{aligned}$ & $\begin{array}{l}0.63 \\
0.26\end{array}$ \\
\hline $\mathrm{PheO}_{\mathrm{D} 2}$ & 1.768 & 0.12 & $\begin{aligned} \text { HOMO } & \rightarrow \text { LUMO } \\
\text { HOMO- } & \rightarrow \text { LUMO+1 }\end{aligned}$ & $\begin{array}{l}0.62 \\
0.27\end{array}$ \\
\hline
\end{tabular}


Table S15. The vertical excitation energies of the lowest excited state (i.e. $\mathrm{Q}_{y}$ ) using gas-phase optimized geometry of the Chlorophyll $a$ computed using DLPNO-STEOM-CCSD, CC2, ADC(2), SOS-CC2, SOS$\mathrm{ADC}(2), \mathrm{SCS}-\mathrm{CC} 2$ and SCS-ADC(2) The geometry in this case is optimized at the PBE/Def2-TZVP level of theory. Corresponding oscillator strengths $(f)$ are also shown. The major contributions in the canonical basis associated with the $\mathrm{Q}_{y}$ state are described. The coordinates for this geometry are provided at the end of this document.

\begin{tabular}{|c|c|c|c|c|}
\hline Method & Energy (eV) & $f$ & Frontier Molecular Orbitals & $\%$ Contribution \\
\hline $\begin{array}{c}\text { DLPNO- } \\
\text { STEOM-CCSD }\end{array}$ & 1.663 & 0.25 & $\begin{aligned} \mathrm{HOMO} & \rightarrow \text { LUMO } \\
\mathrm{HOMO}-1 & \rightarrow \text { LUMO+1 }\end{aligned}$ & $\begin{array}{l}0.72 \\
0.19\end{array}$ \\
\hline $\mathrm{CC} 2$ & 2.146 & 0.25 & $\begin{aligned} \mathrm{HOMO} & \rightarrow \text { LUMO } \\
\mathrm{HOMO}-1 & \rightarrow \text { LUMO+1 }\end{aligned}$ & $\begin{array}{l}0.70 \\
0.16\end{array}$ \\
\hline SOS-CC2 & 2.021 & 0.23 & $\begin{aligned} \mathrm{HOMO} & \rightarrow \text { LUMO } \\
\mathrm{HOMO}-1 & \rightarrow \text { LUMO+1 }\end{aligned}$ & $\begin{array}{l}0.75 \\
0.19\end{array}$ \\
\hline $\mathrm{SCS}-\mathrm{CC} 2$ & 2.064 & 0.24 & $\begin{aligned} \mathrm{HOMO} & \rightarrow \text { LUMO } \\
\mathrm{HOMO}-1 & \rightarrow \text { LUMO+1 }\end{aligned}$ & $\begin{array}{l}0.77 \\
0.18\end{array}$ \\
\hline $\operatorname{ADC}(2)$ & 1.925 & 0.29 & $\begin{aligned} \mathrm{HOMO} & \rightarrow \text { LUMO } \\
\mathrm{HOMO}-1 & \rightarrow \text { LUMO+1 }\end{aligned}$ & $\begin{array}{l}0.74 \\
0.11\end{array}$ \\
\hline SOS-ADC(2) & 1.936 & 0.26 & $\begin{aligned} \mathrm{HOMO} & \rightarrow \text { LUMO } \\
\mathrm{HOMO}-1 & \rightarrow \text { LUMO+1 }\end{aligned}$ & $\begin{array}{l}0.77 \\
0.16\end{array}$ \\
\hline SCS-ADC(2) & 1.947 & 0.28 & $\begin{aligned} \mathrm{HOMO} & \rightarrow \text { LUMO } \\
\mathrm{HOMO}-1 & \rightarrow \text { LUMO+1 }\end{aligned}$ & $\begin{array}{l}0.79 \\
0.15\end{array}$ \\
\hline
\end{tabular}


1. $P_{D 1}$

\begin{tabular}{|c|c|c|c|}
\hline & 00 & 00 & \\
\hline & 2000 & 2000 & 1000 \\
\hline & 000 & -0.675062000 & 000 \\
\hline & 96000 & -0.930 & \\
\hline & 416000 & 0.371020000 & 000 \\
\hline & 6000 & 72000 & 1000 \\
\hline & 2000 & 000 & 000 \\
\hline & 000 & 000 & 000 \\
\hline & 000 & 5000 & 000 \\
\hline & 000 & 000 & 000 \\
\hline & 000 & 000 & 000 \\
\hline & 000 & 000 & -5 \\
\hline & & 000 & 000 \\
\hline & & 000 & 000 \\
\hline & & & \\
\hline & & 00 & \\
\hline & -2.0 & & \\
\hline & & -0 . & \\
\hline & & & \\
\hline & & & \\
\hline & & & \\
\hline & & & \\
\hline & & & \\
\hline & & & \\
\hline & & & \\
\hline & & & \\
\hline & & & \\
\hline & & & \\
\hline & & & \\
\hline & & & \\
\hline & & & \\
\hline & & & \\
\hline & & & 000 \\
\hline & & & 000 \\
\hline & & & \\
\hline E & & & \\
\hline & & & 000 \\
\hline C & & & 000 \\
\hline C & & & 000 \\
\hline $\mathrm{C}$ & & & \\
\hline $\mathrm{C}$ & & & \\
\hline 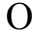 & & & 3000 \\
\hline $\mathrm{C}$ & & & 000 \\
\hline $\mathrm{C}$ & -0.94 & & 000 \\
\hline $\mathrm{C}$ & -0.1 & -0.2 & 000 \\
\hline $\mathrm{C}$ & 00 & 4000 & 45000 \\
\hline $\mathrm{C}$ & 0.39 & -1 & 81000 \\
\hline 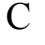 & $-0.56 \mathrm{C}$ & & -2.089473000 \\
\hline 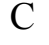 & 533000 & -1.158533000 & 1.2 \\
\hline & 1.102223 & -4.162249000 & -1.68707700 \\
\hline
\end{tabular}




$\begin{array}{lrrr}\mathrm{N} & 0.150668000 & -0.519906000 & 0.012120000 \\ \mathrm{Mg} & 0.787417000 & -1.199088000 & -1.884403000 \\ \mathrm{H} & -1.593067000 & -3.662074000 & -5.329312000 \\ \mathrm{H} & -1.009769000 & -5.279062000 & -4.915586000 \\ \mathrm{H} & -1.364020000 & 4.164735000 & -1.637540000 \\ \mathrm{H} & 0.481549000 & 0.253682000 & 4.270959000 \\ \mathrm{H} & 0.349765000 & -1.477150000 & 4.005600000 \\ \mathrm{H} & -2.314936000 & 4.612484000 & -4.527375000 \\ \mathrm{H} & -2.470206000 & 5.735033000 & -3.056189000 \\ \mathrm{H} & -2.151667000 & -1.339696000 & 4.080028000 \\ \mathrm{H} & -2.076345000 & 0.425138000 & 4.167933000 \\ \mathrm{H} & -1.458426000 & -0.547081000 & 5.508421000 \\ \mathrm{H} & 0.452681000 & -6.481441000 & -3.679642000 \\ \mathrm{H} & 3.565982000 & -8.201122000 & -5.923682000 \\ \mathrm{H} & 3.269699000 & -9.454939000 & -4.657145000 \\ \mathrm{H} & 4.382943000 & -8.076823000 & -4.336094000 \\ \mathrm{H} & -0.092012000 & 0.128887000 & -6.138455000 \\ \mathrm{H} & -1.073893000 & 2.620669000 & -0.318842000 \\ \mathrm{H} & 0.739744000 & -2.869980000 & 2.411686000 \\ \mathrm{H} & 2.240229000 & -2.895762000 & -7.615656000 \\ \mathrm{H} & 2.814281000 & -2.333108000 & -6.019037000 \\ \mathrm{H} & 2.079534000 & -1.178781000 & -7.157276000 \\ \mathrm{H} & -0.431221000 & 2.139405000 & -6.445619000 \\ \mathrm{H} & -0.473863000 & 3.715851000 & -5.626742000 \\ \mathrm{H} & -1.976911000 & 2.822468000 & -5.892988000 \\ \mathrm{H} & -0.828425000 & 2.119285000 & 3.461476000 \\ \mathrm{H} & -2.060827000 & 2.330273000 & 2.203467000 \\ \mathrm{H} & -0.465049000 & 3.057866000 & 2.008495000 \\ \mathrm{H} & 0.677545000 & -6.422478000 & 1.828803000 \\ \mathrm{H} & 1.325476000 & -4.940843000 & 2.590446000 \\ \mathrm{H} & 2.396366000 & -6.079407000 & 1.745880000 \\ \mathrm{H} & 1.267884000 & -4.593403000 & -5.927997000 \\ \mathrm{H} & -0.119998000 & -2.338501000 & -6.950339000 \\ \mathrm{H} & -1.140234000 & -4.800848000 & -6.625450000 \\ \mathrm{H} & 6.946715000 & -1.360860000 & -3.305316000\end{array}$

2. $P_{D 2}$

$\begin{array}{llll}\mathrm{C} & -11.022748000 & 3.671999000 & -1.950795000 \\ \mathrm{H} & -11.272523000 & 4.612080000 & -1.450057000 \\ \mathrm{H} & -11.465349000 & 2.864540000 & -1.351235000 \\ \mathrm{C} & -9.535578000 & 3.487269000 & -2.025157000 \\ \mathrm{~N} & -8.934424000 & 2.340769000 & -1.557392000 \\ \mathrm{H} & -9.428578000 & 1.509355000 & -1.209747000 \\ \mathrm{C} & -7.607336000 & 2.413907000 & -1.788479000 \\ \mathrm{H} & -6.900746000 & 1.638932000 & -1.508996000 \\ \mathrm{~N} & -7.303074000 & 3.564213000 & -2.386263000 \\ \mathrm{C} & -8.498963000 & 4.240367000 & -2.543647000 \\ \mathrm{H} & -8.534807000 & 5.228216000 & -2.995544000 \\ \mathrm{C} & -4.552423000 & 7.622774000 & -6.676039000 \\ \mathrm{C} & -3.182927000 & -1.051278000 & -3.785480000 \\ \mathrm{C} & -4.729078000 & 3.034200000 & 2.882779000 \\ \mathrm{C} & -6.010874000 & 9.287992000 & -2.231040000 \\ \mathrm{~N} & -5.332948000 & 5.114555000 & -4.595771000 \\ \mathrm{C} & -3.606767000 & -2.232829000 & -4.259697000\end{array}$




\begin{tabular}{|c|c|c|c|}
\hline & 0 & 0 & 0 \\
\hline$C$ & -6.126400000 & 8.796863000 & -3764532000 \\
\hline $\mathrm{N}$ & -4.518827000 & 2.400809000 & -3695798000 \\
\hline $\mathrm{O}$ & -6.173567000 & 10.442556000 & .88288300 \\
\hline $\mathrm{C}$ & -7.529239000 & 9.021535000 & -4.272106000 \\
\hline $\mathrm{N}$ & -5.167708000 & 5.877749000 & -1.742130000 \\
\hline $\mathrm{C}$ & -8.980742000 & 10.557227000 & -5.321535000 \\
\hline $\mathrm{C}$ & .744971000 & 26000 & 727000 \\
\hline $\mathrm{C}$ & 47000 & 2000 & $\$ 2000$ \\
\hline $\mathrm{C}$ & 01000 & 1.04 & -1. \\
\hline $\mathrm{C}$ & -4.840103000 & 3000 & 0.5 \\
\hline $\mathrm{C}$ & 1000 & 7000 & 52000 \\
\hline $\mathrm{C}$ & -3.6 & 0.3 & -6. \\
\hline $\mathrm{C}$ & $-3.9^{\prime}$ & 0.3 & 000 \\
\hline $\mathrm{C}$ & -5.2 & 6000 & 1.0 \\
\hline $\mathrm{C}$ & -5.6 & 6.43 & -4.7 \\
\hline $\mathrm{C}$ & -4.4 & 2.2( & -5.0 \\
\hline $\mathrm{C}$ & -4.2 & 1.9 & -0. \\
\hline $\mathrm{C}$ & -5.0 & 6.2 & $-0 .$. \\
\hline $\mathrm{O}$ & -8.4 & 8.2 & -4 \\
\hline $\mathrm{C}$ & -5.7 & 6.7 & -6.2 \\
\hline $\mathrm{C}$ & -3.9 & & -5 . \\
\hline $\mathrm{C}$ & -4.2 & 1.6 & 0. \\
\hline $\mathrm{C}$ & -5.3 & 7.6 & 0000 \\
\hline $\mathrm{O}$ & -7.6 & 10.2 & 2000 \\
\hline $\mathrm{C}$ & -5.7 & & -6.9 \\
\hline $\mathrm{C}$ & -3.7 & & -4 \\
\hline $\mathrm{C}$ & -4.5 & & 000 \\
\hline $\mathrm{C}$ & -5.6 & 8.1 & 8000 \\
\hline $\mathrm{C}$ & -5.2 & 4.4 & 8000 \\
\hline $\mathrm{C}$ & -4.0 & & 3000 \\
\hline $\mathrm{C}$ & -4.6 & & 000 \\
\hline $\mathrm{C}$ & -5.5 & 7.0 & 0000 \\
\hline $\mathrm{N}$ & -4.5 & 3.2 & 1000 \\
\hline $\mathrm{Mg}$ & 00 & 000 & -2 \\
\hline $\mathrm{H}$ & -3. & & -6.5 \\
\hline $\mathrm{H}$ & -4 & & \\
\hline $\mathrm{H}$ & -2. & -1.0 & -3 \\
\hline $\mathrm{H}$ & -3. & & \\
\hline $\mathrm{H}$ & -4 & 2.0 & \\
\hline $\mathrm{H}$ & -4 & -2.3 & -4.9 \\
\hline $\mathrm{H}$ & -3 . & -3 . & -3. \\
\hline $\mathrm{H}$ & & & \\
\hline $\mathrm{H}$ & -6. & 4.7 & 3.2 \\
\hline $\mathrm{H}$ & & 3.3 & 4.2 \\
\hline $\mathrm{H}$ & -5 . & 9.4 & -4.3 \\
\hline $\mathrm{H}$ & -9 . & 10. & -6 \\
\hline $\mathrm{H}$ & -8.977721000 & 77000 & -5.50 \\
\hline $\mathrm{H}$ & -9.7 & 32000 & -4.5 \\
\hline $\mathrm{H}$ & -4.86 & & -7.0 \\
\hline $\mathrm{H}$ & -3.712 & 0.04 & -1.392921000 \\
\hline $\mathrm{H}$ & -4.83 & & \\
\hline $\mathrm{H}$ & & & -8.119033000 \\
\hline $\mathrm{H}$ & -7.87 & 60000 & -6.503684000 \\
\hline $\mathrm{H}$ & & 3.96 & -7.78416900 \\
\hline $\mathrm{H}$ & -3.246953000 & -0.695237000 & -6.589897000 \\
\hline
\end{tabular}




$\begin{array}{ccrc}\text { H } & -2.966642000 & 0.944858000 & -7.222555000 \\ \mathrm{H} & -4.585377000 & 0.230895000 & -7.315392000 \\ \mathrm{H} & -4.553284000 & -0.486424000 & 0.845558000 \\ \mathrm{H} & -4.208665000 & 0.271288000 & 2.411781000 \\ \mathrm{H} & -2.907292000 & 0.045441000 & 1.243720000 \\ \mathrm{H} & -5.949024000 & 9.250742000 & 1.072445000 \\ \mathrm{H} & -4.279839000 & 8.728888000 & 1.302026000 \\ \mathrm{H} & -5.579723000 & 7.722747000 & 1.913884000 \\ \mathrm{H} & -6.690109000 & 7.344443000 & -6.445380000 \\ \mathrm{H} & -5.101600000 & 5.374212000 & -7.766450000 \\ \mathrm{H} & -4.626722000 & 7.947537000 & -7.713862000 \\ \mathrm{H} & -11.490202000 & 3.698130000 & -2.935107000\end{array}$

\section{Chl 1}

$\begin{array}{llll}\mathrm{C} & -10.810443000 & -6.084505000 & -7.523658000 \\ \mathrm{C} & -4.656624000 & -12.511456000 & -6.162534000 \\ \mathrm{C} & -2.086851000 & -6.322250000 & -1.919862000 \\ \mathrm{C} & -9.453513000 & -3.174805000 & -3.548861000 \\ \mathrm{~N} & -8.959229000 & -7.651019000 & -5.258760000 \\ \mathrm{C} & -4.674525000 & -13.639000000 & -6.903597000 \\ \mathrm{C} & -1.784117000 & -7.195385000 & -0.692121000 \\ \mathrm{C} & -10.358640000 & -4.171354000 & -4.381471000 \\ \mathrm{~N} & -6.705894000 & -9.575810000 & -5.245589000 \\ \mathrm{O} & -9.872844000 & -2.086488000 & -3.172643000 \\ \mathrm{C} & -11.527277000 & -4.584377000 & -3.515704000 \\ \mathrm{~N} & -7.117667000 & -5.803083000 & -3.848199000 \\ \mathrm{C} & -13.878907000 & -4.906592000 & -3.428779000 \\ \mathrm{C} & -9.470323000 & -5.381844000 & -4.627178000 \\ \mathrm{C} & -8.969117000 & -9.924217000 & -6.150975000 \\ \mathrm{C} & -4.313763000 & -9.804594000 & -4.749128000 \\ \mathrm{C} & -4.964088000 & -5.400834000 & -2.794230000 \\ \mathrm{C} & -11.974033000 & -8.964765000 & -5.210545000 \\ \mathrm{C} & -7.760084000 & -12.647446000 & -7.092363000 \\ \mathrm{C} & -1.548951000 & -9.020877000 & -3.565779000 \\ \mathrm{C} & -6.300804000 & -2.550577000 & -2.097796000 \\ \mathrm{C} & -9.788603000 & -6.564711000 & -5.265661000 \\ \mathrm{C} & -7.638839000 & -10.330944000 & -5.900839000 \\ \mathrm{C} & -4.071383000 & -8.588707000 & -4.092731000 \\ \mathrm{C} & -6.256777000 & -4.979111000 & -3.127942000 \\ \mathrm{O} & -11.408013000 & -5.018274000 & -2.381801000 \\ \mathrm{C} & -10.985728000 & -6.810167000 & -6.165807000 \\ \mathrm{C} & -7.049995000 & -11.573146000 & -6.346732000 \\ \mathrm{C} & -2.800300000 & -8.212284000 & -3.502791000 \\ \mathrm{C} & -6.899212000 & -3.715977000 & -2.808069000 \\ \mathrm{O} & -12.707684000 & -4.452534000 & -4.150057000 \\ \mathrm{C} & -11.005647000 & -8.354400000 & -6.233663000 \\ \mathrm{C} & -5.710382000 & -11.542247000 & -5.963820000 \\ \mathrm{C} & -3.015336000 & -7.014323000 & -2.859525000 \\ \mathrm{C} & -8.184457000 & -3.832327000 & -3.356419000 \\ \mathrm{C} & -9.559060000 & -8.696746000 & -5.885178000 \\ \mathrm{C} & -5.520788000 & -10.271669000 & -5.272786000 \\ \mathrm{C} & -4.387033000 & -6.628189000 & -3.150955000 \\ \mathrm{C} & -8.248396000 & -5.114500000 & -3.978881000 \\ \mathrm{~N} & -5.014366000 & -7.622715000 & -3.873103000 \\ \mathrm{Mg} & -7.088999000 & -7.844699000 & -4.151969000 \\ & & & \end{array}$




$\begin{array}{llll}\mathrm{H} & -10.851956000 & -4.997637000 & -7.357655000 \\ \mathrm{H} & -11.647910000 & -6.331614000 & -8.193115000 \\ \mathrm{H} & -3.725839000 & -12.295603000 & -5.628967000 \\ \mathrm{H} & -2.511774000 & -5.364438000 & -1.598022000 \\ \mathrm{H} & -1.161982000 & -6.058677000 & -2.444190000 \\ \mathrm{H} & -5.544262000 & -13.958276000 & -7.472008000 \\ \mathrm{H} & -3.796184000 & -14.283376000 & -6.943107000 \\ \mathrm{H} & -1.020703000 & -6.732963000 & -0.056581000 \\ \mathrm{H} & -1.412671000 & -8.184536000 & -0.982845000 \\ \mathrm{H} & -2.690464000 & -7.348193000 & -0.093887000 \\ \mathrm{H} & -10.722467000 & -3.664398000 & -5.287865000 \\ \mathrm{H} & -14.679699000 & -4.201527000 & -3.661185000 \\ \mathrm{H} & -13.683742000 & -4.920643000 & -2.353062000 \\ \mathrm{H} & -14.132024000 & -5.912539000 & -3.787760000 \\ \mathrm{H} & -9.605646000 & -10.659797000 & -6.641167000 \\ \mathrm{H} & -3.453379000 & -10.469661000 & -4.833147000 \\ \mathrm{H} & -4.350703000 & -4.717995000 & -2.206916000 \\ \mathrm{H} & -11.937117000 & -10.062699000 & -5.234122000 \\ \mathrm{H} & -13.007100000 & -8.643927000 & -5.412732000 \\ \mathrm{H} & -11.708532000 & -8.647165000 & -4.191936000 \\ \mathrm{H} & -8.848941000 & -12.544246000 & -7.013838000 \\ \mathrm{H} & -7.481301000 & -13.637873000 & -6.708431000 \\ \mathrm{H} & -7.502316000 & -12.640261000 & -8.164814000 \\ \mathrm{H} & -1.678385000 & -9.985375000 & -3.055882000 \\ \mathrm{H} & -0.708712000 & -8.510230000 & -3.082171000 \\ \mathrm{H} & -1.257318000 & -9.238113000 & -4.605067000 \\ \mathrm{H} & -5.829213000 & -1.863354000 & -2.818794000 \\ \mathrm{H} & -5.532068000 & -2.860943000 & -1.379226000 \\ \mathrm{H} & -7.065265000 & -1.969544000 & -1.564867000 \\ \mathrm{H} & -11.913759000 & -6.427356000 & -5.716163000 \\ \mathrm{H} & -11.276821000 & -8.705484000 & -7.240718000 \\ \mathrm{O} & -8.124328000 & -8.400456000 & -2.453477000 \\ \mathrm{H} & -8.595619000 & -7.657014000 & -1.962026000 \\ \mathrm{H} & -7.684303000 & -8.903141000 & -1.731645000 \\ \mathrm{O} & -9.268408000 & -6.408855000 & -1.095814000 \\ \mathrm{H} & -9.972141000 & -5.841189000 & -1.478310000 \\ \mathrm{H} & -9.361138000 & -6.352022000 & -0.121204000 \\ \mathrm{H} & -9.879590000 & -6.291951000 & -8.051433000\end{array}$

4. $\mathrm{Chl}_{\mathrm{D} 2}$

$\begin{array}{lrcl}\mathrm{C} & 6.415649000 & 7.974332000 & -8.721952000 \\ \mathrm{C} & 0.709841000 & 15.156722000 & -7.053634000 \\ \mathrm{C} & -2.364416000 & 9.044716000 & -3.025333000 \\ \mathrm{C} & 4.975949000 & 5.601465000 & -4.392073000 \\ \mathrm{~N} & 4.531634000 & 9.842713000 & -6.614432000 \\ \mathrm{C} & 0.708470000 & 16.264213000 & -7.819837000 \\ \mathrm{C} & -2.517624000 & 9.688443000 & -1.637451000 \\ \mathrm{C} & 5.878677000 & 6.474945000 & -5.364676000 \\ \mathrm{~N} & 2.461400000 & 11.956535000 & -6.462500000 \\ \mathrm{O} & 5.393793000 & 4.572361000 & -3.877829000 \\ \mathrm{C} & 7.061247000 & 6.985115000 & -4.567327000 \\ \mathrm{~N} & 2.684596000 & 8.234903000 & -4.926269000 \\ \mathrm{C} & 9.410053000 & 7.040598000 & -4.317648000 \\ \mathrm{C} & 4.993508000 & 7.647371000 & -5.749177000 \\ \mathrm{C} & 4.627599000 & 12.055273000 & -7.637674000\end{array}$




\begin{tabular}{|c|c|c|c|}
\hline 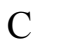 & 0 & 00 & - \\
\hline $\mathrm{C}$ & 000 & 000 & 3000 \\
\hline $\mathrm{C}$ & 009000 & 4000 & -6 \\
\hline $\mathrm{C}$ & 3.626527000 & 29991000 & 2000 \\
\hline $\mathrm{C}$ & -2.605898000 & 11.898438000 & 9000 \\
\hline $\mathrm{C}$ & 1.820419000 & 5.232033000 & 000 \\
\hline $\mathrm{C}$ & 5.325354000 & 000 & 3000 \\
\hline $\mathrm{C}$ & 3.377607000 & 881000 & 0000 \\
\hline $\mathrm{C}$ & 2000 & 0000 & 7000 \\
\hline $\mathrm{C}$ & 00 & & 000 \\
\hline $\mathrm{O}$ & 6.95 & 000 & 000 \\
\hline $\mathrm{C}$ & 6.53 & & 000 \\
\hline $\mathrm{C}$ & 2.88 & 000 & 5000 \\
\hline $\mathrm{C}$ & -1.42 & 10. & 5000 \\
\hline $\mathrm{C}$ & 000 & & -3.6 \\
\hline $\mathrm{O}$ & 8.24 & & -5.0 \\
\hline $\mathrm{C}$ & 6.57 & 000 & 7000 \\
\hline $\mathrm{C}$ & 1.6 & 000 & 8000 \\
\hline $\mathrm{C}$ & -1.3 & & 000 \\
\hline $\mathrm{C}$ & 00 & & -4.2 \\
\hline $\mathrm{C}$ & 5.15 & & 000 \\
\hline $\mathrm{C}$ & & & 000 \\
\hline $\mathrm{C}$ & & & 000 \\
\hline $\mathrm{C}$ & 3.79 & & $-5 .($ \\
\hline $\mathrm{N}$ & 0.7 & 10. & 000 \\
\hline $\mathrm{Mg}$ & 000 & 3000 & 7000 \\
\hline $\mathrm{H}$ & 6.4 & & -8 \\
\hline $\mathrm{H}$ & 7.2 & & \\
\hline $\mathrm{H}$ & -0.0 & 000 & 000 \\
\hline $\mathrm{H}$ & -2.1 & & -2 \\
\hline $\mathrm{H}$ & 00 & & -3 \\
\hline $\mathrm{H}$ & & & -8 \\
\hline $\mathrm{H}$ & -0 & & 00 \\
\hline $\mathrm{H}$ & & & 00 \\
\hline $\mathrm{H}$ & & & -1 . \\
\hline $\mathrm{H}$ & & & -1 \\
\hline $\mathrm{H}$ & & & -6 \\
\hline $\mathrm{H}$ & & & -3 \\
\hline $\mathrm{H}$ & & & \\
\hline $\mathrm{H}$ & 00 & & -4 \\
\hline $\mathrm{H}$ & & & -8 \\
\hline $\mathrm{H}$ & & & \\
\hline $\mathrm{H}$ & & & \\
\hline $\mathrm{H}$ & & & 000 \\
\hline $\mathrm{H}$ & & & 000 \\
\hline $\mathrm{H}$ & & & 000 \\
\hline $\mathrm{H}$ & & & \\
\hline $\mathrm{H}$ & & & 000 \\
\hline $\mathrm{H}$ & & 00 & 000 \\
\hline $\mathrm{H}$ & & 000 & 000 \\
\hline $\mathrm{H}$ & & 000 & 000 \\
\hline $\mathrm{H}$ & -2.84 & 000 & 000 \\
\hline $\mathrm{H}$ & & & -3.362 \\
\hline $\mathrm{H}$ & 00 & & -2.4 \\
\hline $\mathrm{H}$ & 2.446 & 4.98 & -1.937269000 \\
\hline $\mathrm{H}$ & 7.451887000 & 8.532292000 & -6.933375000 \\
\hline
\end{tabular}




$\begin{array}{llll}\mathrm{H} & 6.787282000 & 10.607219000 & -8.765825000 \\ \mathrm{O} & 3.920034000 & 10.804230000 & -3.762742000 \\ \mathrm{H} & 4.208042000 & 11.696258000 & -3.516107000 \\ \mathrm{H} & 4.296514000 & 10.147331000 & -3.079707000 \\ \mathrm{O} & 4.847236000 & 8.982889000 & -2.173282000 \\ \mathrm{H} & 5.022543000 & 9.043072000 & -1.209283000 \\ \mathrm{H} & 5.577982000 & 8.459424000 & -2.572351000 \\ \mathrm{H} & 5.500383000 & 8.122249000 & -9.295080000\end{array}$

\section{Pheop1}

$\begin{array}{llll}\mathrm{C} & -5.779036000 & -14.313517000 & -15.277842000 \\ \mathrm{C} & -4.907337000 & -7.492629000 & -9.022869000 \\ \mathrm{C} & 0.209299000 & -5.409055000 & -14.730193000 \\ \mathrm{C} & -2.797078000 & -12.001538000 & -18.486612000 \\ \mathrm{~N} & -5.257916000 & -11.008703000 & -14.473016000 \\ \mathrm{C} & -5.662823000 & -7.415200000 & -7.911420000 \\ \mathrm{C} & 1.510787000 & -6.227966000 & -14.681531000 \\ \mathrm{C} & -4.001125000 & -12.672822000 & -17.693400000 \\ \mathrm{~N} & -4.895731000 & -8.985857000 & -12.363174000 \\ \mathrm{O} & -2.303641000 & -12.472652000 & -19.505200000 \\ \mathrm{C} & -5.181422000 & -12.911639000 & -18.601436000 \\ \mathrm{~N} & -2.862809000 & -9.695975000 & -15.846541000 \\ \mathrm{C} & -7.010419000 & -11.912076000 & -19.719031000 \\ \mathrm{C} & -4.235829000 & -11.756494000 & -16.502132000 \\ \mathrm{C} & -6.562341000 & -10.793831000 & -12.430886000 \\ \mathrm{C} & -3.507586000 & -7.050514000 & -11.751140000 \\ \mathrm{C} & -1.243064000 & -7.884719000 & -15.961042000 \\ \mathrm{C} & -8.556736000 & -11.848560000 & -14.740246000 \\ \mathrm{C} & -7.018706000 & -9.869842000 & -9.464056000 \\ \mathrm{C} & -1.395306000 & -4.813619000 & -11.980809000 \\ \mathrm{C} & -0.453504000 & -9.411003000 & -18.684038000 \\ \mathrm{C} & -5.222261000 & -11.854905000 & -15.536110000 \\ \mathrm{C} & -5.885278000 & -9.749585000 & -11.793682000 \\ \mathrm{C} & -2.673581000 & -6.920389000 & -12.879798000 \\ \mathrm{C} & -1.817318000 & -9.041075000 & -16.503887000 \\ \mathrm{O} & -5.459552000 & -13.990434000 & -19.106967000 \\ \mathrm{C} & -6.328609000 & -12.895949000 & -15.505877000 \\ \mathrm{C} & -6.099149000 & -9.257547000 & -10.457391000 \\ \mathrm{C} & -1.637838000 & -5.887389000 & -12.984425000 \\ \mathrm{C} & -1.513426000 & -9.759819000 & -17.697598000 \\ \mathrm{O} & -5.884880000 & -11.787863000 & -18.822222000 \\ \mathrm{C} & -7.191881000 & -12.398905000 & -14.319990000 \\ \mathrm{C} & -5.222133000 & -8.192384000 & -10.253510000 \\ \mathrm{C} & -0.977232000 & -6.123641000 & -14.163097000 \\ \mathrm{C} & -2.424971000 & -10.831924000 & -17.728571000 \\ \mathrm{C} & -6.305413000 & -11.334271000 & -13.691176000 \\ \mathrm{C} & -4.475852000 & -8.006660000 & -11.490155000 \\ \mathrm{C} & -1.634470000 & -7.285642000 & -14.762050000 \\ \mathrm{C} & -3.253940000 & -10.754169000 & -16.574788000 \\ \mathrm{~N} & -2.663443000 & -7.745453000 & -13.961062000 \\ \mathrm{H} & -4.558754000 & -9.106430000 & -13.315700000 \\ \mathrm{H} & -3.205736000 & -9.324859000 & -14.948768000 \\ \mathrm{H} & -5.344063000 & -14.331868000 & -14.269382000 \\ \mathrm{H} & -4.959694000 & -14.541861000 & -15.975242000 \\ \mathrm{H} & -3.909515000 & -7.044643000 & -8.992562000\end{array}$




$\begin{array}{crcc}\mathrm{H} & 0.363024000 & -4.473612000 & -14.171267000 \\ \mathrm{H} & 0.001972000 & -5.111231000 & -15.769769000 \\ \mathrm{H} & -6.666932000 & -7.829200000 & -7.844311000 \\ \mathrm{H} & -5.277216000 & -6.920258000 & -7.019610000 \\ \mathrm{H} & 2.369031000 & -5.618159000 & -14.992000000 \\ \mathrm{H} & 1.463381000 & -7.111769000 & -15.332849000 \\ \mathrm{H} & 1.706068000 & -6.578257000 & -13.660074000 \\ \mathrm{H} & -3.644507000 & -13.669761000 & -17.393132000 \\ \mathrm{H} & -7.825242000 & -12.449491000 & -19.223171000 \\ \mathrm{H} & -6.717990000 & -12.441020000 & -20.634310000 \\ \mathrm{H} & -7.312983000 & -10.885683000 & -19.947701000 \\ \mathrm{H} & -7.374262000 & -11.247514000 & -11.863067000 \\ \mathrm{H} & -3.332766000 & -6.336596000 & -10.946110000 \\ \mathrm{H} & -0.410379000 & -7.441405000 & -16.504181000 \\ \mathrm{H} & -8.444292000 & -11.009864000 & -15.443057000 \\ \mathrm{H} & -9.118050000 & -11.495769000 & -13.866922000 \\ \mathrm{H} & -9.157462000 & -12.629503000 & -15.228328000 \\ \mathrm{H} & -7.911102000 & -9.250669000 & -9.291199000 \\ \mathrm{H} & -6.510069000 & -9.962848000 & -8.494975000 \\ \mathrm{H} & -7.355088000 & -10.866362000 & -9.775898000 \\ \mathrm{H} & -0.614426000 & -4.126694000 & -12.325943000 \\ \mathrm{H} & -1.065081000 & -5.213135000 & -11.011004000 \\ \mathrm{H} & -2.310000000 & -4.227961000 & -11.803491000 \\ \mathrm{H} & 0.448447000 & -9.042051000 & -18.177002000 \\ \mathrm{H} & -0.788718000 & -8.614154000 & -19.364654000 \\ \mathrm{H} & -0.192257000 & -10.284212000 & -19.294948000 \\ \mathrm{H} & -6.904628000 & -12.849476000 & -16.446719000 \\ \mathrm{H} & -7.351812000 & -13.208718000 & -13.593782000 \\ \mathrm{H} & -6.531968000 & -15.094958000 & -15.380363000\end{array}$

\section{Pheo 2}

$\begin{array}{lrrl}\mathrm{C} & 1.286223000 & 15.625657000 & -17.037650000 \\ \mathrm{C} & 0.859739000 & 9.331298000 & -10.315170000 \\ \mathrm{C} & -4.929460000 & 7.256077000 & -15.423025000 \\ \mathrm{C} & -1.493314000 & 12.839529000 & -20.223511000 \\ \mathrm{~N} & 0.760150000 & 12.554059000 & -15.966991000 \\ \mathrm{C} & 1.484424000 & 9.596059000 & -9.151202000 \\ \mathrm{C} & -6.318892000 & 7.896633000 & -15.582210000 \\ \mathrm{C} & -0.201586000 & 13.491147000 & -19.554555000 \\ \mathrm{~N} & 0.331210000 & 10.918080000 & -13.581624000 \\ \mathrm{O} & -1.868912000 & 13.100064000 & -21.362053000 \\ \mathrm{C} & 1.036964000 & 13.218693000 & -20.386649000 \\ \mathrm{~N} & -1.871157000 & 11.358117000 & -17.058722000 \\ \mathrm{C} & 2.896754000 & 11.778889000 & -20.662797000 \\ \mathrm{C} & -0.181745000 & 12.946588000 & -18.138267000 \\ \mathrm{C} & 2.198267000 & 12.460921000 & -14.007331000 \\ \mathrm{C} & -1.149931000 & 9.168329000 & -12.689701000 \\ \mathrm{C} & -3.650400000 & 9.721273000 & -16.826775000 \\ \mathrm{C} & 4.009645000 & 12.927778000 & -16.622598000 \\ \mathrm{C} & 3.014077000 & 11.582731000 & -11.117030000 \\ \mathrm{C} & -3.199944000 & 6.868045000 & -12.714293000 \\ \mathrm{C} & -4.210751000 & 10.652380000 & -19.884495000 \\ \mathrm{C} & 0.770151000 & 13.194240000 & -17.165694000 \\ \mathrm{C} & 1.483125000 & 11.564049000 & -13.209506000 \\ \mathrm{C} & -2.113536000 & 9.038890000 & -13.714063000\end{array}$




\begin{tabular}{crcc}
$\mathrm{C}$ & -2.978344000 & 10.691322000 & -17.587368000 \\
$\mathrm{O}$ & 1.446524000 & 13.923493000 & -21.291498000 \\
$\mathrm{C}$ & 1.882655000 & 14.220284000 & -17.267745000 \\
$\mathrm{C}$ & 1.841624000 & 11.097352000 & -11.895673000 \\
$\mathrm{C}$ & -3.118824000 & 7.965582000 & -13.719788000 \\
$\mathrm{C}$ & -3.143991000 & 11.105422000 & -18.944743000 \\
$\mathrm{O}$ & 1.649792000 & 12.064006000 & -20.008648000 \\
$\mathrm{C}$ & 2.816954000 & 13.755969000 & -16.126133000 \\
$\mathrm{C}$ & 0.910616000 & 10.128591000 & -11.525196000 \\
$\mathrm{C}$ & -3.853234000 & 8.134345000 & -14.865712000 \\
$\mathrm{C}$ & -2.070763000 & 11.984315000 & -19.206409000 \\
$\mathrm{C}$ & 1.877033000 & 12.894939000 & -15.292618000 \\
$\mathrm{C}$ & -0.055531000 & 10.017763000 & -12.614347000 \\
$\mathrm{C}$ & -3.270367000 & 9.288980000 & -15.551978000 \\
$\mathrm{C}$ & -1.294041000 & 12.100756000 & -18.019282000 \\
$\mathrm{~N}$ & -2.227498000 & 9.824290000 & -14.818271000 \\
$\mathrm{H}$ & -0.116971000 & 11.044840000 & -14.487128000 \\
$\mathrm{H}$ & -1.572067000 & 11.153798000 & -16.093642000 \\
$\mathrm{H}$ & 0.945818000 & 15.682767000 & -15.992874000 \\
$\mathrm{H}$ & 0.389989000 & 15.773469000 & -17.659902000 \\
$\mathrm{H}$ & 0.208087000 & 8.454367000 & -10.345159000 \\
$\mathrm{H}$ & -5.018959000 & 6.364726000 & -14.785007000 \\
$\mathrm{H}$ & -4.600206000 & 6.886424000 & -16.407143000 \\
$\mathrm{H}$ & 2.097849000 & 10.478280000 & -8.984419000 \\
$\mathrm{H}$ & 1.340113000 & 8.936606000 & -8.296122000 \\
$\mathrm{H}$ & -6.943606000 & 7.278408000 & -16.241013000 \\
$\mathrm{H}$ & -6.259590000 & 8.899479000 & -16.026671000 \\
$\mathrm{H}$ & -6.846652000 & 7.980818000 & -14.624487000 \\
$\mathrm{H}$ & -0.342427000 & 14.579811000 & -19.583054000 \\
$\mathrm{H}$ & 2.742491000 & 11.527151000 & -21.721742000 \\
$\mathrm{H}$ & 3.315884000 & 10.920461000 & -20.127049000 \\
$\mathrm{H}$ & 1.977155000 & 16.441291000 & -17.250773000 \\
$\mathrm{H}$ & 3.576343000 & 12.639280000 & -20.594941000 \\
$\mathrm{H}$ & 3.118546000 & 12.854169000 & -13.574910000 \\
$\mathrm{H}$ & -1.273969000 & 8.497527000 & -11.839668000 \\
$\mathrm{H}$ & -4.486802000 & 9.211970000 & -17.304421000 \\
$\mathrm{H}$ & 4.622369000 & 12.568549000 & -15.784156000 \\
$\mathrm{H}$ & 4.650324000 & 13.528568000 & -17.283192000 \\
$\mathrm{H}$ & 3.661613000 & 12.050460000 & -17.189029000 \\
$\mathrm{H}$ & 3.733955000 & 12.120120000 & -11.747047000 \\
$\mathrm{H}$ & 3.533587000 & 10.743825000 & -10.636349000 \\
$\mathrm{H}$ & 2.704844000 & 12.266079000 & -10.310868000 \\
$\mathrm{H}$ & -2.288221000 & 6.251237000 & -12.746649000 \\
$\mathrm{H}$ & -4.061322000 & 6.214462000 & -12.903874000 \\
& -3.285528000 & 7.248213000 & -11.688114000 \\
$\mathrm{H}$ & -5.886310000 & 10.270547000 & -19.341919000 \\
$\mathrm{H}$ & 2.391165000 & 14.164777000 & -18.244859000 \\
\hline
\end{tabular}


PBE/Def2-TZVP Gas-Phase Optimized Geometry of Chlorophyll $a$

C -6.96955532980476

C -3.97831446364572

C 2.45801151962580

C -2.25026671626436

N -4.58659058760425

H -6.98958954258222

C -4.89233224915220

C 2.52385946996387

C -3.74599807311062

N -3.77223904702334

O -1.87383718468599

C -4.09203904321669

$\mathrm{N}-1.85831276169031$

C -4.82281511907293

C -3.73610606053716

C -5.92528970775784

C -1.92803971175853

C 0.36022880270147

C $\quad-6.79236886599198$

C -6.64031937301997

C 0.94793893748929

C 0.94177079268199

C -4.75622380891666

C -5.05202006781342

C -0.92991324334456

C -0.54813174111192

O -3.93119799304285

C -6.17460823102262

C $\quad-5.35849264033969$

C 0.41389822242334

C -0.32594555069147

O -4.58073709434601

C -6.72225844141453

C -4.22033174139175

C 1.06386186242389

C -1.54635156551351

C $\quad-5.71068747576708$

C -3.22780166128736

C 0.10805657613462

C -2.43920267987864

$\mathrm{N} \quad-1.09128831387975$

Mg -2.82908677152284

H -6.52924865915722

H -8.00731053865120

H $\quad-2.92414538058384$

H 3.08680002802214

H 2.90197812048313

H $\quad-5.96379662293370$

H $\quad-4.57814101692053$

H 3.55867848830001

H 1.92097918095002

H 2.12915290783496

H -4.40195652559290

H $\quad-5.19709578144030$
35.83164086066760

34.71442905049682

37.09786154429551

35.85967478018361

34.88156724656598

36.57850224351265

34.72948689924828

38.62831126608160

35.42120662514685

34.97699286375801

36.02293371624963

34.12740795346006

35.81191110379756

33.21105520941798

35.33027716483542

34.22783557201340

35.53689316425194

36.45692962956236

32.41253494646393

33.80542421905502

36.46968974618806

36.75803224725851

34.94904232325437

34.50967153667435

35.90740357907517

36.22159822221447

33.00906596236503

34.59020996249630

34.33542229852746

36.33553271306462

36.35236474374192

34.38187257126852

33.94328099918019

34.71372501976756

36.58964979459695

36.00789345544521

34.38347673631521

35.10277567822087

36.32086507410688

35.68994099652298

35.90893933672996

35.39254551907499

36.30486166012889

35.55832942094849

34.70714595736468

36.77746790380147

36.63588674622336

34.78007986612220

34.71912524256556

38.96942775066955

38.97661643942445

39.11245008606240

36.21463927293521

33.58757420563515
8.03087168346602

$-0.71308596099324$

3.41015181100734

9.96992474855971

5.84430804701611

7.22343249572018

$-1.70223098938268$

3.53936219159616

9.57471435664959

2.97409194529472

11.11651349952769

10.27977253109801

6.44112915379949

12.32358878357142

8.05963353418130

3.89514612776429

1.44864988062107

5.65072406411389

6.39593124954742

0.87353634555162

0.63596432033778

8.78956480066019

7.20743091331833

2.82490358112479

2.36906362609743

6.68973612285199

9.82885145666293

7.60195831178209

1.42029928321632

2.02406126481293

8.11836227341463

11.51799599260500

6.30815144852252

0.71606414645017

3.21533443017390

8.70421046205845

5.26017815683008

1.71318573719710

4.27691472404557

7.64205750443535

3.73011409984025

4.72337394089593

8.91909448387902

8.27203244496026

$-1.00899440381850$

2.56459371405324

4.30667032059589

$-1.51332031097650$

$-2.74608102450737$

3.68827003164468

4.39010883661435

2.63474380195741

9.96605403960215

13.28076507620918 


$\begin{array}{cccc}\mathrm{H} & -3.89182642716462 & 32.64683241500218 & 12.46618879623649 \\ \mathrm{H} & -5.56498856074647 & 32.55882189634930 & 11.84372442131859 \\ \mathrm{H} & -6.90395466887648 & 33.83114597727975 & 3.62011159735627 \\ \mathrm{H} & -1.64454379926567 & 35.59646372317199 & 0.39704499642482 \\ \mathrm{H} & 1.36294784211267 & 36.78092893583597 & 5.93526832059795 \\ \mathrm{H} & -7.10269209982149 & 31.97218692157744 & 5.43796722506948 \\ \mathrm{H} & -7.51302112794546 & 32.10349047217936 & 7.16722942589882 \\ \mathrm{H} & -5.80946051293792 & 31.99653072793389 & 6.66114446142932 \\ \mathrm{H} & -6.45610328543132 & 33.16376714798754 & -0.00100706944107 \\ \mathrm{H} & -7.31295218976082 & 34.61434054515752 & 0.54286687581539 \\ \mathrm{H} & -7.18576188492248 & 33.21368464240687 & 1.62087530442276 \\ \mathrm{H} & 2.01964951049906 & 36.70858030111329 & 0.64196068664663 \\ \mathrm{H} & 0.43520479229202 & 37.27068036564848 & 0.07955188329943 \\ \mathrm{H} & 0.81647434269838 & 35.54170567440227 & 0.05803091212039 \\ \mathrm{H} & 0.79472655720226 & 36.84120984694055 & 9.87300989840401 \\ \mathrm{H} & 1.30604949455989 & 37.72643943992408 & 8.41269141892819 \\ \mathrm{H} & 1.74220520217878 & 36.02263437339533 & 8.60959099626279 \\ \mathrm{H} & -6.16636801731903 & 33.86158602379247 & 8.42730063398050 \\ \mathrm{H} & -7.72274823890209 & 34.33636840986109 & 6.06599257797070\end{array}$

